\title{
ON A CONJECTURE OF KOCH ${ }^{1}$
}

\author{
R. P. HUNTER
}

Let $X$ be a topological space. We recall that $D$, a subset of $X$ is called a $C$-set if any continuum which meets $D$ and its complement must contain $D$.

Let $S$ be a continuum which is a topological semigroup with identity 1 , and let $H$ denote the maximal subgroup of $S$ containing 1 . It is well known that $H$ exists and is compact. The following four conjectures have been raised and shown to be equivalent by Koch, $[2] .^{2}$

(1) The unit is not a weak-cutpoint.

(2) $S$ is aposyndetic at any point with respect to 1 .

(3) The identity component of $H$ is not a nontrivial $C$-set.

(4) 1 belongs to no nontrivial $C$-set.

We give here an affirmative answer to these conjectures. (We assume, of course, that $S$ is not a group.)

THEOREM. Let $G$ be a compact invariant subgroup of $H$ such that $H / G$ is a Lie group. Then $S$ contains a continuum $M$ such that $M$ meets $H$ and the complement of $H$ and such that $M \cap H \subseteq G$.

Proof. We consider $H$ as a transformation group of $S$ in the obvious way. Letting $H^{\prime}=H / G$ and letting $S^{\prime}$ denote the space of orbits of $G$, we may consider $H^{\prime}$ as a transformation group of $S^{\prime}$. Finally letting $S^{\prime \prime}$ denote the space of orbits under $H$ itself, we have the following diagram.

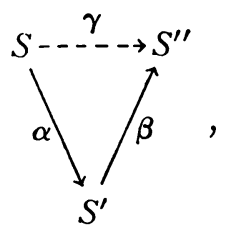

where $\gamma=\alpha \beta$, and $\alpha, \beta$, and $\gamma$, are all canonical mappings, as $S^{\prime \prime}$ may also be considered as the space of orbits of $S^{\prime}$ under $H^{\prime}$. Since the decompositions defined by the sets $\{x H\}$ or by $\{x G\}, x \in S$, are con-

Received by the editors March 2, 1960.

1 The author holds an S. H. Moss Postdoctoral Fellowship.

${ }^{2} \mathrm{Koch}$ in [2] had affirmed this conjecture in case $S$ was either homogeneous or one dimensional. 
tinuous, the mappings $\gamma$ and $\alpha$ are both open. It follows that $\beta$ is open also.

Now $H^{\prime}$ is a compact Lie group of transformations acting on a compact connected space $S^{\prime}$. Gleason [1, Theorem 3.3] has shown that there is a closed neighborhood $N$ of $1^{\prime}$ such that the orbit of any point of $N$ meets a certain set $L$ in precisely one point. That is to say there is a closed neighborhood $N$ and a closed set $L$ such that $n H^{\prime} \cap L$ is a single point for each $n \in N$, i.e. a local cross section at $1^{\prime}$. $\left(1^{\prime}\right.$, of course, denotes the identity of $S^{\prime}$.)

Now let $\Delta=\left.\beta\right|_{L}$. It is easy to see that $\Delta$ is a homeomorphism between $L$ and $\beta(N)$. Letting $N^{0}$ denote the interior of $N$, we note that $\beta\left(N^{0}\right)$ is an open set about the point $\beta\left(1^{\prime}\right)=\gamma(1)$. Since $S^{\prime \prime}$ is compact and connected there is a nondegenerate continuum $P$ which contains $\gamma(1)=\beta\left(1^{\prime}\right)$ and which is contained in $\beta(N)$. Indeed, let $P$ be the closure of the component of $\beta\left(N^{0}\right)$ which contains the point $\beta\left(1^{\prime}\right)$. It is well known that $P$ must meet the boundary of $\beta\left(N^{0}\right)$. Clearly then, $\Delta^{-1}(P)$ is a continuum which meets $H^{\prime}$ at only $L \cap H^{\prime}$ and of course meets the complement of $H^{\prime}$. Let $\Delta^{-1}(P)=Q$. Since $\alpha$ is an open mapping it follows from Theorem 1.5 of [3] that if $K$ is any component of $\alpha^{-1}(Q)$ then $\alpha(K)=Q$. Letting $K$ be such a continuum we see that $K$ meets the complement of $H$ and is such that $K \cap H$ is contained in some $\alpha^{-1}\left(n^{\prime}\right)$ where $n^{\prime} \in N$. That is to say $K \cap H$ is nonvacuous and is contained in some $y G$ for some $y \in S$, and certainly we must have $y \in H$ since $S-H$ is an ideal of $S$. The desired continuum may be taken as $\bar{y} K$ where $\bar{y}$ is the inverse of $y$ in $H$. For if $k \in K \cap y G$ then $\bar{y} k \in G$ and if $k \notin K \cap y G$ then $k \notin H$ and $\bar{y} k \notin H$ since $S-H$ is an ideal.

CoROLlaRy. If $S$ is a compact connected semigroup with identity then the identity component of $H$ is not a $C$-set.

Proof. One has only to note that there are arbitrarily small invariant subgroups such as $G$ such that $H / G$ is a Lie group.

\section{BIBLIOGRAPHY}

1. A. M. Gleason, Spaces with a compact Lie group of transformations, Proc. Amer. Math. Soc. vol. 1 (1950) p. 35.

2. R. J. Koch, Note on weak cutpoints in clans, Duke Math. J. vol. 24 (1957) p. 611.

3. G. T. Whyburn, Interior transformations on compact sets, Duke Math. J. vol. 3 (1957) p. 371.

OXFORD UNIVERSITY AND

THE UNIVERSITY OF GeORGIA 